\title{
AECHMEA AENIGMATICA (BROMELIACEAE; BROMELIOIDEAE) UNA NUEVA ESPECIE DEL ESTADO DE OAXACA, MÉXICO
}

\author{
Ana Rosa López-Ferrari, Adolfo Espejo-Serna, Jacqueline Ceja-Romero y \\ Aniceto Mendoza-Ruiz
}

Universidad Autónoma Metropolitana-Iztapalapa, División de Ciencias Biológicas y de la Salud, Departamento de Biología, Apdo. postal 55-535, 09340 México, D.F. México. arlf@xanum.uam.mx

\section{RESUMEN}

Se describe e ilustra Aechmea aenigmatica López-Ferrari, Espejo, Ceja et A. Mendoza, una nueva especie de Oaxaca, México. El taxon aquí descrito se compara con A. lueddemanniana (K. Koch) Mez y con A. mexicana Baker, especies con características similares, de las cuales difiere por la presencia de sépalos simétricos y de pétalos sin apéndices basales. Se incluyen claves para la identificación de los taxa de Aechmea presentes en Oaxaca, así como para todos los representantes del subgénero Podaechmea.

Palabras clave: Aechmea, Bromeliaceae, México, Oaxaca, Podaechmea.

\section{ABSTRACT}

Aechmea aenigmatica López-Ferrari, Espejo, Ceja et A. Mendoza, a new species from Oaxaca, Mexico is described and illustrated. The new taxon is compared with $A$. lueddemanniana (K. Koch) Mez and A. mexicana Baker, species with similar characteristics, from which it differs by the presence of symmetrical sepals and petals without basal appendages. Identification keys for all species of Aechmea present in Oaxaca, as well as for all species of subgenus Podaechmea are included.

Key words: Aechmea, Bromeliaceae, Mexico, Oaxaca, Podaechmea.

El género Aechmea (Bromelioideae, Bromeliaceae) agrupa cerca de 240 especies neotropicales (Luther, 2008), que habitan principalmente en Sudamérica, ocho de las cuales crecen en México (Espejo-Serna y López-Ferrari, 1994; Espejo-Serna 
et al., 2004). Mez (1896) dividió formalmente por primera vez al género en seis subgéneros y actualmente se reconocen ocho (Smith y Downs, 1979), mismos que Smith y Kress $(1989,1990)$ elevaron a la categoría de género, sin que su propuesta haya tenido aceptación general.

La taxonomía de Aechmea es sumamente compleja y no hay acuerdos generales acerca de su delimitación tanto genérica como infragenérica, resultando además evidente en diversos trabajos relacionados con el tema (Wendt, 1997; Izquierdo y Piñero, 1998; Horres et al., 2000; Faria et al., 2004; Brown y Leme, 2005; Betancur y Salinas, 2006; Horres et al., 2007; Aguirre-Santoro y Betancur, 2008; Schulte y Zizka, 2008; Faria et al., 2010; Sass y Specht, 2010) que no es un género monofilético y que lo mismo ocurre con sus relaciones infragenéricas. Sin embargo, no se cuenta hasta el momento con una clasificación alternativa a la propuesta de Smith y Downs (1979), por lo que ésta sigue siendo una referencia obligada para los distintos trabajos sobre el género.

Durante uno de los recorridos botánicos realizados en el marco del proyecto Flora Bromeliológica Mexicana, recolectamos plantas vivas de una especie poco común de Aechmea. Un estudio detallado de dicho material nos permitió establecer que se trataba de una especie nueva para la ciencia. La inflorescencia compuesta con las flores pediceladas y polísticas y los sépalos apiculados, ubican al nuevo taxon en el subgénero Podaechmea (Mez, 1896; Smith y Downs, 1979), integrado hasta ahora por cuatro especies, dos de las cuales, Aechmea lueddemanniana (K. Koch) Mez y A. mexicana Baker, se distribuyen en nuestro país, la tercera es endémica de Perú (Aechmea ferruginea L. B. Sm.) y la restante se conoce de Panamá (Aechmea haltonii $\mathrm{H}$. Luther). Con este nuevo hallazgo, el subgénero queda integrado por cinco especies, incluyendo al taxon que a continuación describimos.

Anteriormente también se incluían dentro del subgénero a Aechmea mcvaughii L. B. Smith y a Aechmea tuitensis P. Magaña \& E. J. Lott, sin embargo fueron reubicadas por Read y Baensch (1994) en el género Ursulaea, decisión cuestionada por Izquierdo y Piñero (1998) con base en su trabajo con aloenezimas, pero que hasta el momento se ha mantenido. En trabajos filogenéticos recientes sobre la taxonomía de las Bromelioideae (Schulte et al., 2009; Sass y Specht, 2010), se han incluido representantes de los distintos subgéneros. En el caso de Podaechmea, han sido utilizadas Aechmea lueddemanniana y A. mexicana, las cuales han quedado como parte de un conglomerado mayor en el que se encuentran también los géneros Androlepis, Hogenbergiopsis y Ursulaea, resultando evidente la necesidad de realizar más estudios con la finalidad de encontrar sinapomorfias que permitan esclarecer las relaciones entre los distintos taxa de la subfamilia. 
Aechmea aenigmatica López-Ferrari, Espejo, Ceja et A. Mendoza, sp. nov. Fig. 1(A-H).

Aechmeae mexicanae Baker foliis glaucis longioribus, sepalis in symmetria utrinque congruentibus, petalis albis exappendiculatis et baccis maturis flavis differt.

Hierbas rupícolas rosuladas, en flor de hasta $1.4 \mathrm{~m}$ de alto, las rosetas de tipo tanque, de 1.6 a 1.9 m de diámetro, cortamente caulescentes; rizomas conspicuos, repentes, de ca. $5 \mathrm{~cm}$ de diámetro; hojas numerosas, las vainas verdes, oblongas, de 18 a $28 \mathrm{~cm}$ de largo, de 9 a $20 \mathrm{~cm}$ de ancho, enteras, densa pero inconspicuamente translúcido-lepidotas en ambas superficies; las láminas de color verde glauco, oblongas y cintiformes, en forma de $\mathrm{U}$ en corte transversal, ascendentes a erectas, de 36 a $110 \mathrm{~cm}$ de largo, de 6.5 a $14 \mathrm{~cm}$ de ancho, densa pero inconspicuamente translúcidolepidotas en ambas superficies, finamente serradas en el margen, apiculadas y pungentes en el ápice. Inflorescencia terminal, erecta, paniculada, dos veces dividida, más corta que las rosetas, de 65 a $80 \mathrm{~cm}$ de alto, el pedúnculo cilíndrico, de 40 a $50 \mathrm{~cm}$ de largo, de 1.7 a $2 \mathrm{~cm}$ de diámetro, verde, glabro a esparcidamente lepidoto; brácteas del pedúnculo rojas, oblongo-elípticas, de 15 a $16 \mathrm{~cm}$ de largo, de 4 a $4.5 \mathrm{~cm}$ de ancho, enteras en el margen, excepto en la porción apical que es eroso-denticula$\mathrm{da}$, densa pero inconspicuamente lepidotas en ambas superficies, apiculadas y pungentes en el ápice, imbricadas, más largas que los entrenudos y cubriendo totalmente al pedúnculo; panícula cónica en contorno general, de 30 a 35 cm de largo, el raquis cilíndrico, verde, glabro a esparcidamente lepidoto, de 4 a $12 \mathrm{~mm}$ de diámetro, los racimos primarios 14 a 16, subsésiles a cortamente pediculados, ascendentes, de 2 a $8.5 \mathrm{~cm}$ de largo, los inferiores con dos a tres racimos secundarios en la base, el terminal de ca. $4.5 \mathrm{~cm}$ de largo; brácteas primarias rojas, de 2 a $14 \mathrm{~cm}$ de largo, de 1 a $3 \mathrm{~cm}$ de ancho, densamente lepidotas en ambas superficies, enteras a ligeramente erosas en el ápice, las basales similares a las del pedúnculo, las apicales lineares a filiformes; brácteas florales blancas en la base, rojas a rosadas hacia el ápice, angostamente triangulares, de 3.5 a $6.5 \mathrm{~mm}$ de largo, de ca. $0.5 \mathrm{~mm}$ de ancho, enteras, glabras pero con un mechón de escamas en la base; flores polísticas, divaricadas a ascendentes, más o menos apretadamente dispuestas, de 5 a 30 por racimo, actinomorfas, pediceladas, los pedicelos verdes, cilíndricos, glabros, lineares, más largos que las brácteas florales, de 6 a $9 \mathrm{~mm}$ de largo; sépalos verdes con el ápice rosado, triangulares, simétricos, de 4 a $5 \mathrm{~mm}$ de largo, de ca. $4 \mathrm{~mm}$ de ancho, glabros, con dos alas semicirculares hialinas en los márgenes, apiculados en el ápice, el apículo 

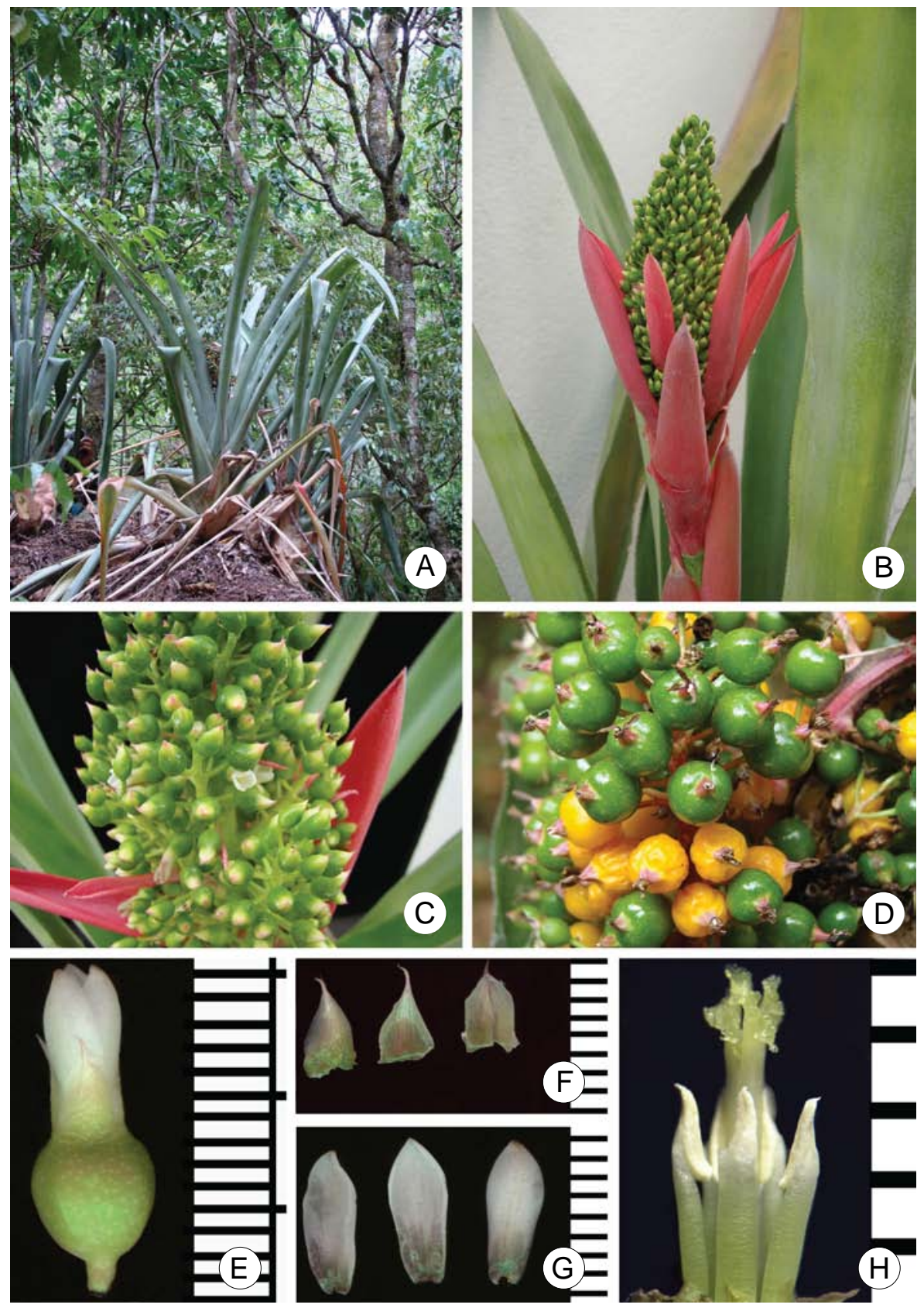

Fig. 1. A. hábito; B. inflorescencia; C. detalle de la inflorescencia con flores en antesis; D. frutos; E. flor; F. sépalos; G. pétalos; H. estambres y estilo. 
de 1 a 1.5 mm de largo, persistentes en el fruto; pétalos blancos, elípticos a oblongoelípticos, de 8 a $9.5 \mathrm{~mm}$ de largo, de 3.5 a $4 \mathrm{~mm}$ de ancho, redondeados en el ápice, glabros, sin apéndices basales; estambres iguales, los filamentos blancos, laminares, rectangulares, de ca $3.2 \mathrm{~mm}$ de largo, las anteras amarillas, dorsifijas, sagitadas, de ca. $1.5 \mathrm{~mm}$ de largo; ovario verde, con poros apreciables como puntos blanquecinos, globoso a semi-globoso, de 4 a $5 \mathrm{~mm}$ de diámetro, glabro, estilo blanco, linear, de ca. $4 \mathrm{~mm}$ de largo, estigmas tres, verde-amarillentos, de ca. $1 \mathrm{~mm}$ de largo, del tipo simple erecto (tipo I sensu Brown \& Gilmartin, 1984) espiralados y papilosos en la parte interna. Bayas globosas, de 8 a 10 mm de diámetro, verdes con puntos blancos (en los poros) cuando jóvenes, amarillas cuando maduras, glabras; semillas anaranjadas a pardas o rojizas, oblongas, rectas a algo curvadas, de 2.7 a $3.7 \mathrm{~mm}$ de largo, de ca. $0.8 \mathrm{~mm}$ de ancho, reticuladas.

Tipo: México, Oaxaca, distrito de Miahuatlán, municipio de San Jerónimo Coatlán, 16.7 km después de Soledad Piedra Larga, rumbo a San Jerónimo Coatlán, 16¹1'30" N, 9658'18" W, ca. 1600 m, bosque mesófilo de montaña, junto al arroyo, colectada en fruto 7.III.2009, cultivada y prensada en flor 7.XII.2009, J. Ceja, A. Espejo, A. R. López-Ferrari y A. Mendoza R. 2005 (holotipo: UAMIZ; isotipo IEB).

Distribución y hábitat: Aechmea aenigmatica se conoce únicamente del municipio de San Jerónimo Coatlán, donde crece sobre rocas, formando colonias pequeñas dentro del bosque mesófilo de montaña (Fig. 1A) a una altitud cercana a $1600 \mathrm{~m}$ s.n.m. Florece de noviembre a enero y fructifica de enero a abril.

Etimología: El epíteto específico hace referencia a las características excepcionales de la especie que la diferencian de los otros taxa del subgénero Podaechmea: los sépalos simétricos (Fig. 1F) y los pétalos de color blanco sin apéndices internos basales (Fig. 1G). Además, la ausencia de apéndices en los pétalos (Brown y Terry, 1992) la distingue de todos los demás miembros conocidos del subgénero Podaechmea.

En el Cuadro 1 comparamos las características distintivas del nuevo taxon con los de las otras dos especies de Podaechmea presentes en México: A. lueddemanniana (K. Koch) Mez y A. mexicana Baker (Espejo-Serna et al., 2004; Smith y Downs, 1979).

Con este nuevo taxon, el número de especies del género Aechmea que crecen en el estado de Oaxaca (Espejo-Serna et al., 2007) se incrementa a siete. A continua- 
Acta Botanica Mexicana 95: 1-9 (2011)

Cuadro 1. Características distintivas de las especies de Podaechmea presentes en México.

\begin{tabular}{llll}
\hline & A. lueddemanniana & A. mexicana & A. aenigmatica \\
\hline $\begin{array}{l}\text { Hábito } \\
\text { Color de la lámina } \\
\text { foliar }\end{array}$ & epífita & epífita & rupícola \\
Sépalos & verde con líneas & verde & verde glauco \\
Pétalos & asimétricos & asimétricos & simétricos \\
& rosados a púrpuras, & lilas a magentas, con & blancos, sin \\
& con apéndices & apéndices basales & $\begin{array}{l}\text { apéndices basales } \\
\text { internos }\end{array}$ \\
Fruto maduro & oblongo, lepidoto y & ovoide, lepidoto y & $\begin{array}{l}\text { globoso, glabro y } \\
\text { amarillo }\end{array}$ \\
\hline
\end{tabular}

ción presentamos claves de identificación para todos los representantes de Aechmea presentes en Oaxaca, así como para los taxa del subgénero Poadaechmea descritos hasta ahora.

Clave para identificar las especies de Aechmea presentes en Oaxaca

1 Flores (y frutos) pedicelados.

2 Frutos maduros azules; lámina foliar de 3.5 a $6.5 \mathrm{~cm}$ de ancho; sépalos de $3 \mathrm{a}$ $5 \mathrm{~mm}$ de largo A. lueddemanniana

2 Frutos maduros blancos o amarillos; lámina foliar de (5-)6.5 a 11(-16) cm de ancho; sépalos de 4 a 7 mm largo.

3 Pétalos rosados a púrpuras, con dos apéndices basales internos; frutos maduros ovoides y blancos; sépalos asimétricos; ovarios y frutos lepidotos .. A. mexicana

3 Pétalos blancos; sin apéndices basales internos, frutos maduros globosos y amarillos; sépalos simétricos; ovarios y frutos glabros .... A. aenigmatica

1 Flores (y frutos) sésiles o casi sésiles.

4 Sépalos de 3.4 a $3.8 \mathrm{~cm}$ de largo; plantas terrestres

A. magdalenae

4 Sépalos de 0.3 a $1.2 \mathrm{~cm}$ de largo; plantas epífitas.

5 Brácteas florales imbricadas, de $1.5 \mathrm{a} 1.8 \mathrm{~cm}$ de largo .... A. tillandsioides

5 Brácteas florales no imbricadas, de 3 a $8 \mathrm{~mm}$ de largo.

6 Inflorescencias ramificadas, paniculadas; plantas grandes, de 70 a 200 $\mathrm{cm}$ de alto A. bracteata 
6 Inflorescencias simples, racemosas; plantas pequeñas, de 30 a $60 \mathrm{~cm}$ de alto

A. nudicaulis

Clave para identificar las especies del subgénero Podaechmea

1 Sépalos simétricos; pétalos blancos, sin apéndices internos basales; frutos maduros amarillos A. aenigmatica

1 Sépalos asimétricos; pétalos rosados, purpúreos o blancos en la base y rosadocerúleos en el ápice, con un par de apéndices basales internos; frutos maduros azules a blancos.

2 Plantas densamente estrellado-ferrugíneo-lepidotas; sépalos rojos; pétalos blancos en la base y rosado-cerúleos en el ápice; plantas conocidas sólo de Perú A. ferruginea

2 Plantas blanco-adpreso-lepidotas o punctulado-pardo-lepidotas, sépalos verdes; pétalos rosados a purpúreos.

3 Espinas del margen foliar de 3 a $8 \mathrm{~mm}$ de largo, pardas oscuras; brácteas del pedúnculo conspicuamente espinosas en el margen; plantas conocidas únicamente de Panamá

A. haltonii

3 Espinas del margen foliar de 1 a $2 \mathrm{~mm}$ de largo, verdes a pardas; brácteas del pedúnculo enteras a diminuta e inconspicuamente serradas en el margen.

4 Frutos oblongos, de 11 a $15 \mathrm{~mm}$ de largo por 3 a $5 \mathrm{~mm}$ de diámetro, azules en la madurez; láminas foliares de 3.5 a $6.5 \mathrm{~cm}$ de ancho

A. lueddemanniana

4 Frutos ovoides, de 15 a $17 \mathrm{~mm}$ de largo por 7 a $8 \mathrm{~mm}$ de diámetro, blancos en la madurez; láminas foliares de 6 a $16 \mathrm{~cm}$ de ancho

A. mexicana

\section{AGRADECIMIENTOS}

Deseamos expresar nuestro sincero agradecimiento a Walter Till, Jerzy Rzedowski y Graciela Calderón de Rzedowski por la lectura crítica del manuscrito así como por sus valiosos comentarios al mismo. Las fotografías que ilustran el trabajo fueron tomadas por Aniceto Mendoza Ruiz y Adolfo Espejo Serna. 


\section{LITERATURA CITADA}

Aguirre-Santoro, J. y J. Betancur. 2008. Sinopsis del género Aechmea (Bromeliaceae) para Colombia. Caldasia 30(2): 265-288.

Betancur, J. y N. R. Salinas. 2006. The Pseudaechmea (Bromeliaceae: Bromelioideae) twilight. Caldasia 28: 157-164.

Brown, G. K. y A. J. Gilmartin. 1984. Stigma structure and variation in Bromeliaceae Neglected taxonomic characters. Brittonia 36(4): 364-374.

Brown, G. K. y R. G. Terry. 1992. Petal appendages in Bromeliaceae. Amer. J. Bot. 79: 1051-1071.

Brown, G. K. y E. M. C. Leme. 2005. The re-establishment of Andrea (Bromeliaceae; Bromelioideae), a monotypic genus form Southeastern Brazil threatened with extinction. Taxon 54: 63-70.

Espejo-Serna, A. y A. R. López-Ferrari. 1994. Bromeliaceae In: Las monocotiledóneas mexicanas. Una sinopsis florística. 1. Lista de referencia, parte III. Consejo Nacional de Flora de México, A.C., Universidad Autónoma Metropolitana Iztapalapa, Comisión Nacional para el Conocimiento y uso de la Biodiversidad. México, D.F. 73 pp.

Espejo-Serna, A., A. R. López-Ferrari, I. Ramírez-Morillo, B. K. Holst, H. E. Luther y W. Till. 2004. Checklist of Mexican Bromeliaceae with notes on species distribution and levels of endemism. Selbyana 25: 33-86.

Espejo-Serna, A., A. R. López-Ferrari, N. Martínez-Correa y V. A. Pulido-Esparza. 2007. Bromeliad flora of Oaxaca, Mexico: Richness and distribution. Acta Bot. Mex. 81: 71-147.

Faria, A. P. G., T. Wendt y G. K. Brown. 2004. Cladistic relationships of Aechmea (Bromeliaceae, Bromelioideae) and allied genera. Ann. Missouri Bot. Garden 91: 303-319.

Faria, A. P. G., T. Wendt y G. K. Brown. 2010. A revision of Aechmea subgenus Macrochordion (Bromeliaceae) based on phenetic analysis of morphological variation. Bot. J. Linnean Soc. 162: 1-27.

Horres, R., G. Zizka, G. Kahl y W. Weising. 2000. Molecular phylogenetics of Bromeliaceae: evidence from trnL(UAA) intron sequences of the chloroplast genome. Plant Biol. 2: 306-315.

Horres, R., K. Schulte, K. Weising y G. Zizka. 2007. Systematics of Bromelioideae (Bromeliaceae) - evidence from molecular and anatomical studies. Aliso 23: 27-43.

Izquierdo, L. Y. y D. Piñero. 1998. Allozyme divergence among four species of Podaechmea s. l. and the status of Ursulaea (Bromeliaceae, Bromelioideae). Pl. Syst. Evol. 213: 207-215.

Luther, H. 2008. An alphabetical list of bromeliad binomials. 11a. ed. The Bromeliad Society International. Sarasota, Florida. 110 pp.

Mez, C. 1896. Bromeliaceae. In: De Candolle, C. Monographiae Phanerogamarum 9: 1-990. Masson y Cie. Paris.

Sass, Ch. y Ch. D. Specht. 2010. Phylogenetic estimation of the core Bromelioids with an emphasis on the genus Aechmea (Bromeliaceae). Mol. Phylogenet. Evol. 55: 559-571. 
Schulte, K. y G. Zizka. 2008. Multi locus plastid phylogeny of Bromelioideae (Bromeliaceae) and the taxonomic utility of petal appendages and pollen characters. Candollea 63: 209-225.

Schulte, K., M. H. Barfuss y W. Zizka. 2009. Phylogeny of Bromelioideae (Bromeliaceae) inferred from nuclear and plastid DNA loci reveals the evolution of the tank habit within the subfamily. Mol. Phylogenet. Evol. 51(2): 327-339.

Smith, L. B. y R. J. Downs. 1979. Bromelioideae (Bromeliaceae). Fl. Neotrop. Monogr. 14(3): 1493-2142.

Smith, L. B. y W. J. Kress. 1989. New or restored genera of Bromeliaceae. Phytologia 66(1): 70-79.

Smith, L. B. y W. J. Kress. 1990. New genera of Bromeliaceae. Phytologia 69(4): 271-274.

Wendt, T. 1997. A review of the subgenus Pothuava (Baker) Baker of Aechmea Ruiz \& Pav. (Bromeliaceae) in Brazil. Bot. J. Linnean Soc. 125: 245-271.

Recibido en mayo de 2010.

Aceptado en octubre de 2010. 